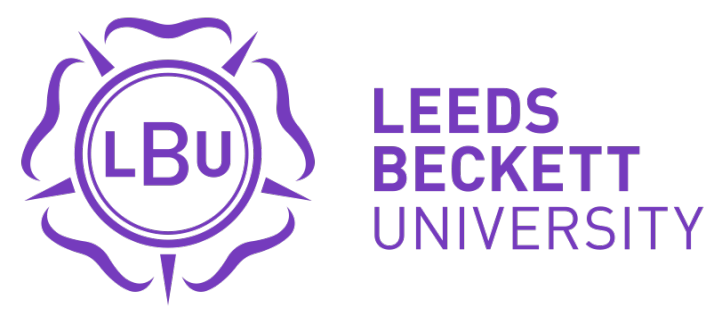

Citation:

Nabaskues-Lasheras, I and Usabiaga, $\mathrm{O}$ and Lozano-Sufrategui, $\mathrm{L}$ and Drew, KJ and Standal, ØF (2019) Sociocultural processes of ability in Physical Education and Physical Education Teacher Education : A systematic review. European Physical Education Review. ISSN 1356-336X DOI: https://doi.org/10.1177/1356336X19891752

Link to Leeds Beckett Repository record:

https://eprints.leedsbeckett.ac.uk/id/eprint/6576/

Document Version:

Article (Accepted Version)

The aim of the Leeds Beckett Repository is to provide open access to our research, as required by funder policies and permitted by publishers and copyright law.

The Leeds Beckett repository holds a wide range of publications, each of which has been checked for copyright and the relevant embargo period has been applied by the Research Services team.

We operate on a standard take-down policy. If you are the author or publisher of an output and you would like it removed from the repository, please contact us and we will investigate on a case-by-case basis.

Each thesis in the repository has been cleared where necessary by the author for third party copyright. If you would like a thesis to be removed from the repository or believe there is an issue with copyright, please contact us on openaccess@leedsbeckett.ac.uk and we will investigate on a case-by-case basis. 


\title{
Sociocultural processes of ability in Physical Education and Physical Education Teacher Education: A systematic review
}

\author{
Itsaso Nabaskues-Lasheras \\ Department of Physical Education and Sports, University of the Basque Country, Spain \\ Oidui Usabiaga \\ Department of Physical Education and Sports, University of the Basque Country, Spain \\ Lorena Lozano-Sufrategui \\ School of Sport, Leeds Beckett University, UK \\ Kevin J Drew \\ School of Sport, Leeds Beckett University, UK \\ Øyvind Førland Standal \\ Faculty of Teacher Education and International Studies, Oslo Metropolitan University, \\ Norway
}

\begin{abstract}
The aim of this study is to systematically review the empirical evidence about the sociocultural processes of ability in Physical Education (PE) and PE Teacher Education (PETE) between 2004 and 2019. Content analysis of 17 included articles demonstrated that all the studies took place in PE, but none were conducted in PETE. Qualitative methodologies were common, underpinned by a variety of theoretical approaches, such as those focused on social structures, discourses, power relations and pedagogical approaches that (re)produce social constructions of ability. Our analysis resulted in four main themes: structuring elements of the field, the able student in PE, (mis)- educational consequences and practical implications. In considering findings from this study, three reflections are presented: (a) there is a clear gap in PETE literature about the sociocultural process of ability in PE; (b) understanding ability as movement literacy could be useful in PE; and (c) future research exploring how ability is socially constructed by different groups of people is needed. PETE programmes should incorporate pedagogical practices in their curriculums directed to challenge students' views about ability, enabling them at the same time to provide more habitable learning environments in their future practice.
\end{abstract}

Keywords

Ability, sociocultural, Physical Education, Physical Education Teacher Education, systematic review 


\section{Introduction}

Since the implementation of inclusive education programmes in western societies (Coates, 2012), the topic of inclusion in Physical Education (PE) has become a central theme in educational research (Overton et al., 2017; Penney et al., 2018). In the area of PE, inclusion relates to the construction of habitable learning environments where all students - regardless of gender, (dis)ability, social class and ethnicity - can experience agency, success and joy through bodily movement (Standal, 2015). Echoing Standal (2015), the philosophy of inclusion in movement contexts involves: (a) respecting and celebrating individual differences; (b) fair and equitable distribution of its benefits; and (c) viewing each pupil as an individual, instead of a collective group. However, in practice, social inequalities still prevail in PE (e.g. Beltra' n-Carrillo and Dev'1s-Dev'1s, 2019; Svendby, 2016), and it seems that there is still work to do to achieve a more inclusive learning environment through both the curriculum and pedagogical practice (Standal, 2015).

According to Evans (2004), promoting inclusion and equity in PE is challenging due to two reasons. First, the prevailing notion of ability in PE is unhelpful, as it is too simplistic, decontextualized and overly focused on ability in a biological sense. Second, there is limited engagement with philosophical thinking around the notion of ability. For Evans (2004), ability is not a neutral concept, but something that nurtures experiences of inequity and exclusion in PE through the reproduction of dominant health and sport discourses that have permeated the field of PE and PE Teacher Education (PETE). In this way, certain body dispositions - differently accessible for students depending on various social characteristics (e.g. gender, class, ethnicity, dis/ability and/or sexuality) - become legitimized, while others are neglected and/or rejected. Drawing on some of Bourdieu's (1990) well-founded theoretical tools - habitus, field and capital - Evans (2004) provided an alternative understanding of ability, where abilities are dynamic and 'embodied social constructs, meaningful only in their display and are always inevitably defined relationally with reference to values, attitudes and mores prevailing within a discursive field' (Evans, 2004: 100). Since Evans reconceptualized the term in 2004, the critical exploration of sociocultural pro- cesses of ability has become an important topic of conversation amongst sociocultural theorists (e.g. Evans, 2014; Flintoff et al., 2008; Wright and Burrows, 2006) and empirical scholars (e.g. Wilkinson et al., 2013). In particular, they are interested in understanding how particular social structures conceptualize and reproduce what is regarded as ability, as well as its educational consequences. To illustrate, Wilkinson et al. (2013) undertook a systematic appraisal to explore how ability is socially constructed within PE. Their findings highlight the negative consequences experienced by those learners who do not bring 'adequate'

dispositions to PE. Further, they found that PE teachers who have an archaic understanding of ability can (re)produce students' inequi- table access to different forms of recognition (capital) such as high grades or positive interactions with agents in the field. To conclude, the authors emphasized the urgent need to challenge tra- ditional and notably narrow conceptions of ability in PE, and the importance of negotiating valuable embodied dispositions (habitus) in educational contexts. This would involve a reconsi- deration of the personal characteristics - either acquired and/or learned based on individuals' class and culture - that are configured as desirable by powerful agents in the field, and thus, are per- ceived as abilities (Evans, 2004). 
Wilkinson et al.'s (2013) findings have made a significant contribution to understandings of ability in PE and the educational consequences of narrow conceptions of ability, and their research has underpinned the development of effective and inclusive practices and equity agendas (Evans and Davies, 2017). However, their work focuses solely on PE, and they only included research informed by the work of Evans (2004). Additionally, there was limited discussion about the practical implications resulting from challenging popular and commonsense conceptions of ability.

In light of the above limitations, this study aims to systematically review the key literature in this area to address the gaps identified in Wilkinson et al.'s (2013) work, with a particular focus on PETE. The reasons for this are twofold: (a) it is an appropriate context to challenge some of the unquestioned internal beliefs held by many future educators (Brown, 2005); and (b) PETE pro- vides an ideal space for generating critical debate on how future educators might deal with the differences children bring to PE, which is an imperative issue for the successful implementation of inclusion (Evans and Penney, 2008). In other words, PETE has the potential to challenge deep- seated beliefs and pedagogies that may be perpetuated in PE (Walton-Fisette et al., 2018). Importantly, given that most students who enter PETE programmes do so with an already con- structed sporting habitus (Brown, 2005), the need to endorse critical reflection and challenge taken-for-granted assumptions about ability is clear. In line with Walton-Fisette et al. (2018), providing future educators with opportunities to reveal, understand and reconstruct their own values and beliefs is key to move beyond understandings of what it means to be able in PE.

Following Evans (2004), the current study is underpinned by a range of theoretical perspectives to scrutinize sociocultural processes of ability in PE. In doing this, its scope is broader than the one provided by Wilkinson et al. (2013). This is a useful contribution as it can facilitate a process of compatibility across different discourse communities — that is, 'a knowledge building process in which knowledge claims are tested against their compatibility (or non-compatibility) with other knowings' (Tinning, 2015: 677). Finding connections across different fields could strengthen the claims made in relation to the sociocultural construction of ability in PE. Furthermore, it may also provide PE teachers with tools to appropriately deal with the rich, diverse and dynamic set of abilities that children bring to school.

Additionally, this study will explore the practical implications of a socially constructed perspective of ability (i.e. drawing on Evans' ideas about ability in PE) suggested in the literature to reconceptualize the notion of ability in PE and PETE. It is important that those working in PETE are aware of alternative conceptions of ability to challenge the problems associated with notably narrow essentialist notions of ability (Evans, 2004). To do so, the practical implications collated in this review will help educators develop strategies to engage students in critical reflection. In doing this, PE teachers will be better equipped to promote joyful and accessible movement experiences for all (Barber, 2018), which may, in turn, contribute to lifelong engagement in the movement culture.

Methods 
A systematic literature review methodology was used with the following aims: (a) to systematically review the empirical body of evidence offering insights into the sociocultural processes of ability in PE and PETE from 2004 to 2019; (b) to identify the theoretical frameworks that have been used in these studies; and (c) to critically analyze the practical implications resulting from conceptualizing ability in ways that are more inclusive and equitable within PE. For the purpose of our review, we adopted a systematic process underpinned by some elements of the evidence-based Preferred Reporting Items for Systematic Reviews and Meta-Analyses (PRISMA) framework (Moher et al., 2009), such as the use and application of the inclusion and exclusion criteria, data extraction, analysis, synthesis and reporting of the results. These processes are explained in detail in the following sections.

\section{Initial search}

As indicated in Figure 1, the following international electronic databases were searched in October 2017: SportDiscus, ERIC, PsycInfo and Scopus. These databases are the most common in this field of study. Searches included a number of key words and terms, grouped into four search categories, including: (a) 'ability' OR 'talent' OR 'capability' OR 'skill' OR 'movement capability' OR 'physicality' AND (b) 'physical education' OR 'sport education' OR 'movement education' OR 'physical education teacher education' OR 'higher education' AND (c) 'conception' OR 'under- standing' OR 'construction' OR 'notion' OR 'legitimization' AND (d) 'sociocultural' OR 'Evans' OR 'Bourdieu' OR 'physical capital'. While the first three search categories were searched in the titles and abstracts, the last search category was searched in the full texts. These terms were identified as the most relevant for our searches after reviewing the literature related to the social construction of ability. Other key concepts such as habitus, field, embodied experience or power were not searched for, as these are background concepts that Evans (2004) drew on when developing the concept of ability.

Each database search included only empirical peer-reviewed journal articles published in English, with abstracts available. Our first database search was limited to the years 20042017. This is because since the publication of Evans' (2004) study, a significant number of relevant studies have been undertaken to explore the sociocultural construction of ability in PE. Our searches resulted in 194 outputs, which were imported into Mendeley Reference Manager for advanced screening.

\section{Article selection}

From the imported references, duplicates were removed (n 76). The title and the abstract of the remaining papers (n 118) were then read by the first author and included or excluded based on these inclusion criteria: (a) the sociocultural processes of ability as key topic; (b) PE or PETE as context; and (c) full text available. This resulted in 95 articles being discarded. At this stage of the screening process, we were left with 23 potential articles. We screened the reference lists of these papers and found seven additional studies that met the inclusion criteria ( $\mathrm{n}$ 30). Three authors discussed the eligibility of studies with regards to the inclusion criteria throughout the screening process, and decisions about including or excluding articles 
were made when all three authors were in agreement. As a result of these eligibility discussions, the number of publications was reduced to 16 .

Not all the included articles focused on sociocultural processes of ability. Some articles merged ability construction with other concepts (e.g. physicality and legitimate movement) or topics (e.g. experiences of disability). Given the closeness of these topics to our research aims, these studies were also included. With the aim of keeping this systematic review up to date, the search was undertaken again in April 2019, and one article meeting the inclusion criteria was included for analysis.

\section{Data analysis}

All the included articles were analyzed using descriptive and content analysis. The latter was achieved following Hennink et al. (2011) and Saldan a (2009). First, each study was read and summarized by the main author in order to reduce data by identifying and coding relevant findings that addressed the research questions. After initial coding, codes were contrasted, compared and categorized based on their meaning and links between codes. This inductive process allowed construction of thematic clusters and categories. Deductive reasoning was also used throughout coding and categorizing, as this process was influenced by Evans' (2004) work. After reviewing the themes, these were labelled and grouped into major categories. Exploring links between these categories allowed for the development of a conceptual understanding of the entire data set. The established categories and codes were then revised and refined, according to the research ques- tions. Finally, to ensure the quality of the analysis process, codes and categories were discussed between the authors until consensus was reached. Following Grant and Booth (2009), the key characteristics of the studies were extracted and input into Table 1. Content analysis revealed four key themes: (a) structuring elements of the field; (b) the able student in PE; (c) (mis)educational consequences; and (d) practical implications. These themes are explained in the results section.

\section{Results}

The following section presents the synthesis of the results. This is followed by a discussion of the findings, recommendations for future research and a conclusion where we summarize key findings and highlight implications for policy and practice.

\section{General overview}

As shown in Table 1, the majority of studies were qualitative (n 16), and one used mixed methods (Hunter, 2004). All studies took place in PE contexts, but none in PETE. Studies were predominantly conducted in the UK (6), Australia (5), Sweden (4), the Netherlands (2) and the USA (2). Research questions in all articles can be summarized as follows: What does it mean 'to be able' in PE?; How are notions of ability produced and reproduced through pedagogical practices; and What are the educational consequences of the ways ability is produced and reproduced through practice? To answer these questions, the reviewed articles 
focused on three key areas of inquiry: (a) PE teachers' understandings of ability; (b) students' experiences of PE; and (c) official texts such as curriculum guidelines.

A number of theoretical frameworks were identified in the studies. The most widely used framework was Bourdieu's habitus, field and capital, and these were often combined with Evans' (2004) alternative notion of ability (Fitzgerald, 2005; Hay and lisahunter, 2006; Hay and Mac- donald, 2010a, 2010b; Koca et al., 2009; Redelius et al., 2009; Tiden et al., 2015) and Shilling's contribution to the idea of the body as a powerful source for physical capital (Croston and Hills, 2017; Evans et al., 2015; Fitzgerald, 2005; Hay and lisahunter, 2006; Hay and Macdonald, 2010a; Hills, 2007; Redelius and Hay, 2009; Tiden et al., 2015). Redelius and Hay (2009) drew on Bourdieu's and Shilling's theories as well as Bernstein's. For Bernstein, assessment, curriculum and pedagogy are three inter-related parts of a message system. In this system, assessment is recognized as a powerful tool in defining what legitimized knowledge means in PE settings. However, Bourdieu's ideas have also been used in combination with critical pedagogy and post-structuralist feminism (Hunter, 2004). Only the studies by Van Amsterdam et al. (2012) and Van Doodewaard et al. (2018) diverged from the approaches identified above. These two studies drew on Foucault's concept of disciplinary power, and were framed within a feminist viewpoint that served to elucidate techniques of categorization and normalization over bodily differences. A further study was underpinned by Weedon's post-structuralist feminist perspective to uncover and challenge prevailing and unquestioned discourses and power relations (Domangue and Solmon, 2012). Finally, Bernstein's concepts of vertical and horizontal pedagogical approaches (Evans and Penney, 2008) and pedagogical devices (Svennberg, 2017) were used to understand how knowl- edge around ability is coded in the PE curriculum, as well as how teachers transform knowledge requirements of movement into pedagogical practice. Findings, therefore, indicate that a variety of frameworks have been employed - sometimes in isolation and at other times in combination - to explore structures, discourses, power relations and pedagogical approaches that shape under- standing of ability in PE.

Structuring elements of the field

Macro context: discourses and official guidelines. The macro context involves policy makers, governors and the broader PE community. Dominant discourses in this context were powerful and con- straining, and contributed to the reproduction of taken-for-granted and narrow versions of PE habitus, including: normativity and categorization of the body (Domangue and Solmon, 2012; Evans et al., 2015; Hunter, 2004; Van Amsterdam et al., 2012), performance culture (Fitzgerald, 2005; Hay and lisahunter, 2006; Hills, 2007; Redelius et al., 2009), hegemonic masculinity (Fitzgerald, 2005; Hay and Macdonald, 2010b; Hunter, 2004), health and fitness (Hunter, 2004) and the positivist paradigm (Tiden et al., 2015).

The macro discourse permeates the field of PE and its discourse(s) are reflected in central structuring elements, such as official assessment guidelines and the curriculum. With regards to assessment guidelines, Tiden et al. (2015) explored the efficacy of 10 different PE assessment tools. The authors found that none of these tools met established criteria, as the tools only measured a limited number of traditional sport-related techniques, which were performed in de- contextualized contexts. Echoing Domangue and Solmon (2012), Tiden et al. (2015) alerted the PE community to the powerful impact assessment tests can have on the 
social construction of ability in PE, as these determine the movement dispositions that are and are not - exchangeable for capital. Thus, assessment is a powerful system that influences students' learning, expectations and beliefs (Redelius and Hay, 2009). However, only pupils who possess valuable dispositions have access to different forms of recognition, whereas others are constrained by their personal histories and power relations, which, in turn, reproduce the unequal distribution of learning opportunities. As a consequence, it is acknowledged that assessment processes can trigger experiences of alienation and symbolic violence for those students who are unable to meet the expectations of specific bodily performances (Redelius et al., 2009).

Similarly, the curriculum itself can also have an impact on assumptions and ideals about valuable forms of physical capital in PE (Evans and Penney, 2008; Hay and Macdonald, 2010a; Koca et al., 2009). To illustrate, Evans and Penney (2008) found two contrasted forms of pedagogy in their analysis of two PE curriculum texts: (a) the horizontal or competencybased pedagogical model; and (b) the vertical or performance-based pedagogical model. Each of these models communicates different assumptions about learning, ability and power relations in the field. While the former values and recognizes body and ability differences, the latter measures and ranks students' abilities based on normativity and principles of differentiation. The authors argued that most current curriculum texts are based on vertical pedagogical models, which are also supported by a political climate that supports a culture of performance and early differentiation.

Agents in the micro context. Structuring elements in the micro context, 'reproducing the game' (Hunter, 2004: 180) involved unaware actions and attitudes of both PE teachers and students (Evans et al., 2015). Regarding students, 'high ability' peers - commonly male sportspersons - were identified as significant reproducers of ability. Harmful practices, such as excluding class- mates within games, teasing or openly ranking peers' bodies (Hills, 2007), allowed them to maintain privileged positions while undermining other students' experiences in PE (Fitzgerald, 2005; Hay and lisahunter, 2006; Hay and Macdonald, 2010a; Hunter, 2004; Koca et al., 2009). Nevertheless, previous authors have also acknowledged that the complicity of students with limited access to capital contributes to the preservation of educational inequities embedded in PE contexts (Hay and lisahunter, 2006; Hunter, 2004).

Most studies identified PE teachers as the most influential mediators in defining what constituted the legitimated habitus in the playing field (Hay and lisahunter, 2006; Hay and Macdonald, 2010b). In fact, educators reinforced the traditional ideology of ability (Fitzgerald, 2005; Hay and Macdonald, 2010a, 2010b) through implicit messages and the impact of power relations on ped- agogical practices (Van Amsterdam et al., 2012; Van Doodewaard et al., 2018). Hay and lisahunter (2006) explored PE teachers' educational practices and found that the powerful messages they communicated were grounded in their own personal experiences within the movement culture. Broader discourses of ability were drawn on by PE teachers; however, it was evident that a dis- course of natural ability prevailed in their descriptions of ability in PE (Croston and Hills, 2017; Svennberg, 2017).

The able student in PE 
Valuable attributes for recognition. The structuring elements outlined above contributed to the def- inition of what 'to be able' means in PE. In the current context, ability in PE is related to the attributes possessed by students and how these fit within existing discourses (Evans and Penney, 2008). In other words, to be a 'good' PE student, pupils must have resources that match the PE habitus as anticipated at each period (Hay and Macdonald, 2010a; Koca et al., 2009). Moreover, students' ability to access capital in PE depends on their previous experiences of practicing sport- related techniques embedded in assessment standards (Hay and lisahunter, 2006; Svennberg, 2017; Tiden et al., 2015).

Specifically, the 'able' student was the one who possessed idealized physicality and attitudinal dispositions informed by sport, performance and hegemonic dogmas that celebrated and valued masculine-related dispositions (Hay and Macdonald, 2010b; Hunter, 2004; Van Doodewaard et al., 2018). Physicality was contingent on dual attributes. It entailed the expression of a masculine and athletic corporeality (Hunter, 2004; Koca et al., 2009; Redelius and Hay, 2009). It also demon- strated physical potentiality in relation to high levels of fitness (Hunter, 2004) and motor skills (Fitzgerald, 2005; Hay and Macdonald, 2010b; Hunter, 2004; Koca et al., 2009; Redelius et al., 2009) during the team-sports commonly played in PE (Evans et al., 2015; Hills, 2007; Redelius and Hay, 2009).

These attributes facilitated the acquisition of physical capital, which was the most valued resource, and could be exchanged for other forms of capital (e.g. social and cultural capital). This, in turn, influenced further participation and enjoyment of PE (Hills, 2007; Koca et al., 2009). Additionally, physical capital could also be gained through participation in afterschool sports clubs (Hay and Macdonald, 2010a; Redelius and Hay, 2009) and through involvement in elite sport (Hay and Macdonald, 2010b).

Alternatively, attitudinal dispositions, whilst appreciated to a lesser extent than corporeal features, were an essential element of a good student for PE teachers (Redelius and Hay, 2009). These included the capacity to display male-associated conducts such as aggressive and com- petitive behaviors (Hay and Macdonald, 2010b; Hunter, 2004), to have a positive attitude towards the subject (Redelius et al., 2009), to show enthusiasm for sports (Hay and Macdonald, 2010a), to demonstrate effort (Hunter, 2004) and to exhibit leader qualities implicitly accessible only for 'astute' performers (Redelius and Hay, 2009). Thus, acceptable forms of embodiment worked to rank PE pupils based on the dispositions they brought to school (Hay and lisahunter, 2006). This selective process was further dependent on the attributes identified below.

Intersectionality matters. Intersectional theory, which originated in Black feminism (e.g. Hooks, 1982), explores the complex relationships between different socially constructed axes of identity, such as class, race, gender, sexuality and disability. In doing this, it aims to make 'visible the multiple positioning that constitutes everyday life and the power relations that are central to it' (Phoenix and Pattynama, 2006: 187). As indicated in Table 1, some of the papers included in this review have focused on how social constructions of ability in PE affect students' experiences, by focusing on either gender (Domangue and Solmon, 2012; Redelius et al., 2009), social class (Hay and Macdonald, 2010a) or disability (Evans et al., 2015; Fitzgerald, 2005), whereas others have explored pupils' lived experiences by addressing the interrelationships between different axes of oppression (Hay and lisahunter, 2006; Hills, 2007; Hunter, 2004; Koca et al., 2009; Van Doo- dewaard et al., 2018). 
In particular, gender, sexuality, disability, social class and ethnicity have been identified as determinant factors on learners' possibilities to be recognized as able. This means that attributes of social order - grounded in binary and hierarchical thinking - influence the eligibility processes of students' abilities in PE - that is, what 'counts' as ability in PE (Domangue and Solmon, 2012; Evans and Penney, 2008). These were reproduced through vertical pedagogical models displaying discourses that were more likely to be embedded by students with particular bodies and tastes (Evans and Penney, 2008). In some cases, educators attempted to compensate for existing inequalities to access learning among pupils with different backgrounds (Svennberg, 2017). Yet, their actions were inconsistent with regards to official assessment criteria.

\section{(Mis)educational consequences}

Experiences of difference(s). Acquisition of physical capital was not equally accessible for all stu- dents, as some constructed categories (e.g. male, white, healthy, slim, fit and ablebodied) were judged to be more admirable and desirable than others (Van Amsterdam et al., 2012; Van Doo- dewaard et al., 2018). Consequently, those being slim, physically mature, talented at ball games and able to exhibit male-associated attributes continued to occupy the most significant positions in the field. These students were given a greater opportunity to successfully display sporting tech- niques (Hay and Macdonald, 2010b; Koca et al., 2009; Redelius and Hay, 2009; Svennberg, 2017). Nevertheless, a divergent reality was experienced by peers whose access to capital was restricted due to the impossibility to meet the demands of the field. These students were excluded and deemed to occupy peripheral positions in PE (Hay and lisahunter, 2006; Hills, 2007; Koca et al., 2009). This picture was repeatedly evoked in the reviewed articles (e.g. Domangue and Solmon, 2012; Fitzgerald, 2005; Hay and lisahunter, 2006; Hay and Macdonald, 2010a; Hills, 2007; Hunter, 2004; Koca et al., 2009), which made explicit the symbolic violence suffered by 'non-able' students. These experiences negatively affected students' motivation and willingness to learn and engage in movement culture in and outside of PE. This meant that their learning and individual development was restricted (Hay and lisahunter, 2006; Svennberg, 2017; Tiden et al., 2015), often by significant others (Domangue and Solmon, 2012; Van Amsterdam et al., 2012). These experiences hindered the students' self-esteem, hopes for achievement (Hay and Macdo- nald, 2010a), self-perception of their bodies as (un)desirable (Van Doodewaard et al., 2018) and self-identification as able (Fitzgerald, 2005; Hay and lisahunter, 2006; Koca et al., 2009). Their sense of physicality was also damaged, as bodies that do not meet normative standards are seen as deviant (Van Amsterdam et al., 2012) and presumed to be inappropriate to perform the movements required in PE (Koca et al., 2009). As a result, some learners' embodied subjectivity - habitus - was constrained (Domangue and Solmon, 2012; Hay and lisahunter, 2006; Hunter, 2004; Van Amsterdam et al., 2012) and overlooked (Hay and Macdonald, 2010a). Persistent experiences of symbolic violence led particular groups of students to become disempowered and excluded from PE (Fitzgerald, 2005), which reinforced a sense of otherness (Domangue and Solmon, 2012).

Closing spaces for inclusion agendas. The available discourses in PE closed spaces for recognizable forms of movement possibilities and diversity among learners (Hunter, 2004; Koca et al., 2009; Svennberg, 2017). In other words, while certain habitus and practices were 
accepted and sup- ported, others were noticeably rejected. Hence, the effectiveness of PE to meet inclusion and equity agendas is arguable (Evans et al., 2015; Redelius and Hay, 2009). In particular, gender inequities for the acquisition of valuable attributes in PE (e.g. high grades and access to precious resources) are evident (Hay and Macdonald, 2010b). Microcontext agents, such as PE teachers and students, nurture and sustain these inequities with their stereotypical beliefs about males' and females' abilities (Van Amsterdam et al., 2012), as well as their assumptions about what is valuable in PE (Redelius et al., 2009).

Interestingly, while a biological understanding of gender differences was dominant among students, notions of ability as a sociocultural process started to emerge too (Domangue and Solmon, 2012).

Counterproductive learning in PE. As we have stated earlier in this paper, there is a prevalence of discursive spaces based on powerful sociocultural trends in PE, which critically diminishes its educational purpose (Evans and Penney, 2008). For instance, it turns out that only a few sport- related activities were legitimated (Koca et al., 2009; Svennberg, 2017). Consequently, students rarely distinguished PE from sports (Hunter, 2004). More importantly, difficulties with articulating what was being learned and valued as legitimated movement among students (Hay and lisahunter, 2006) and teachers (Svennberg, 2017) made explicit that PE continued to be more about doing and being instead of knowing (Redelius et al., 2009).

As a consequence, justification of the subject in the school curriculum was questioned. Some of the embedded learning was considered counter-productive (Tiden et al., 2015) for other goals associated with PE, such as lifelong participation in movement culture (Hay and lisahunter, 2006). As Redelius et al. (2009: 258) have stated, 'many pupils learn [through PE] they are not good enough'.

\section{Practical implications}

The following theme encompasses practical implications identified to overcome existing chal- lenges in relation to sociocultural processes of ability in PE and PETE. A call for active agents in the field. Practical implications were mostly directed towards PE educators and professional development programmes for teachers (Hay and Macdonald, 2010b; Redelius and Hay, 2009). These recognize the vital role teachers have in disrupting and rethinking the field (Fitzgerald, 2005). In order to confront doxa - the taken-for-grantedness of the social world - in PE (Hunter, 2004), reflective practice is considered essential as a way of overcoming deep-rooted unquestioned discourses of normalization and categorization that communicated discriminatory knowledge about the body (Fitzgerald, 2005; Van Amsterdam et al., 2012; Van Doodewaard et al., 2018). The aim of promoting reflective practice among PE teachers was twofold: (a) to encourage educators to critically (re)consider the role performance codes embedded in PE teachers' daily practices play in generating an understanding of ability and learning in the field; and (b) to incite reflection upon the extent to which discourses and pedagogical approaches stimulate an (un)equal desire to learn among students in movement education (Evans and Penney, 2008; Hay and Mac- donald, 2010b). Hence, the need to elucidate PE teachers' discourses of ability was considered a key task to confront taken-for-granted beliefs (Fitzgerald, 2005; Hay and Macdonald, 2010b; Hunter, 2004; Svennberg, 2017; Van Doodewaard et al., 2018). 
More specific implications for PE teachers were associated with challenges related to assessment, curriculum and pedagogy. In relation to assessment, the need to incorporate assessment for learning practices and to broaden the abilities to be assessed was suggested (Tiden et al., 2015). Fitzgerald (2005) and Hunter (2004) also argued that educators should support learners' ability to critically reflect upon how students are differently positioned and recognized by agents in the micro context. Moreover, it is important to ensure consistency between the abilities that are valued and those stated in the syllabus (Redelius et al., 2009; Svennberg, 2017).

Concerning pedagogy, more horizontal (Evans and Penney, 2008), cooperative (Hills, 2007) and participatory pedagogical approaches are needed (Croston and Hills, 2017). These are more likely to positively influence PE students' experiences, regardless of their (physical) abilities. Moreover, the impact of embodied experience in activities that require new skills (e.g. adapted sports) has been acknowledged as a way to overcome students' traditional notions about (dis)- ability in PE (Evans et al., 2015). Curriculum-related suggestions implied a shift in the content and purposes for PE away from a multi-activity model (Redelius et al., 2009; Svennberg, 2017), as well as the incorporation of more inclusive activities and teaching strategies to deal with inclusion, justice and equity agendas (Hay and Macdonald, 2010b; Hills, 2007; Hunter, 2004; Van Amsterdam et al., 2012).

Similarly, implications for PE students included critical reflection upon how students come to be positioned differently in the field and what their contribution is on the sociocultural processes of ability 'that affords some people [ .. . ] capital not possible for others' (Hunter, 2004: 189). This task might assist students to develop a more inclusive standpoint within movement contexts (Hills, 2007). To achieve this, teachers are encouraged to challenge students' deep-constructions of knowledge (Domangue and Solmon, 2012; Van Doodewaard et al., 2018). In a similar vein, PE teacher educators are called to include marginalized PE students' life experiences into their teaching to provoke dialogue among future teachers about ongoing inequity in the field (Domangue and Solmon, 2012).

To conclude, a wider call invited the broader field of PE (Croston and Hills, 2017) to challenge oppressive ideologies in order to move beyond categorical thinking around the body and ability in PE, which is central to recognize multiple embodied subjectivities, and to meet the needs of a higher number of students (Hunter, 2004). More generally, Koca et al. (2009) encouraged the school community to critically reflect upon how exclusionary practices in educational settings might impact on learners' capacity to celebrate diversity in society. This can be achieved by constructing more inclusive and democratic learning spaces in schools.

\section{Discussion}

This review offers a synthesis of empirical evidence about the sociocultural processes of ability in PE and PETE, including the theoretical frameworks that underpin the literature in this area. In 
doing this, we have critically analyzed what is suggested to be a more appropriate way to think about ability in order to meet principles of inclusion in PE (Standal, 2015).

It is evident from our findings that an understanding of ability is still an obvious source of negative and exclusionary experiences, which, in turn, is an obstacle to deal with challenges of inclusion and social justice in PE (Evans, 2004; Fitzgerald, 2012). In line with earlier research (Wiltshire et al., 2017), experiences of inclusion/exclusion depend on the amount of physical and social capital students possess and on how meaningful PE is for students. That is, $\mathrm{PE}$ continues to generate constraining consequences, because only some unquestioned qualities grounded on macro-context ideologies are implicitly rewarded (Beltra'n-Carrillo and Dev'1s-Dev'1s, 2019; Brown, 2005). A biological understanding of ability (Hay, 2005) and corrective devices, such as ableist normative notions of physicality (Standal, 2015), act as oppressive power structures that constrain some pupils from having a positive experience in PE. This is because abilities are ranked based on limited but identifiable attributes (Flintoff et al., 2008; Wright and Burrows, 2006). Thus, recognition and meaningful learning is available only for a few students that fit into constraining ideologies of performance, sports and healthism (Beltra' n-Carrillo and Dev'1s-Dev'1s, 2019). Yet, this is inconsistent with the educational values of PE (Standal and Aggerholm, 2016). Dominant discourses in PE are problematic, as the PE environment fails to match many learners' capacities and interests. They fail to provide all students with opportunities to acquire practical knowledge and curtail meaningful embodied experiences. Consequently, mis-educational experiences in PE are common, which might limit children's lifelong participation in movement culture. These outcomes work against the aims and objectives of the subject in the school curriculum (Standal, 2015; Wright and Burrows, 2006). These findings are also relevant to the closely related context of extra-curricular school sports, where similar conceptions of ability and gender have been identified (e.g. Wilkinson and Penney, 2016).

As previously highlighted, none of the articles included in this review took place in PETE. It has been more than a decade since Evans articulated ability as a social construct in PE; however, little remains known about the social construction of ability in PETE. One explanation could be that articles that used similar concepts but not ability have been excluded from this review (Nyberg et al., 2019), which is a limitation of this study. Other closely related issues such as social justice (e.g. Walton-Fisette and Sutherland, 2018) or dominant discourses about the body (e.g. Wrench and Garrett, 2015) have progressively gained attention in PETE literature. However, little research has focused specifically on the sociocultural construction of ability and how this construction intersects with gender, race, ethnicity or sexuality. This may mean that debates around socio- cultural processes of ability are absent in these contexts, which also minimizes possibilities for change. Thus, despite its advocacy, debates about understandings of ability in PE struggle to find a place within PETE (Walton-Fisette et al., 2018). A reason for this might be that the ethos in PETE continues to operate in a traditional manner, by nurturing hegemonic (Serra, 2016), normative and sportoriented professional knowledge of a technical-rational kind (Dowling, 2011). Simultaneously, sociocultural and social justice issues are left unaddressed (Felis-Anaya et al., 2017). Consequently, the effectiveness of PETE programmes towards inclusion and diversity is ques- tionable (Coates, 2012).

In light of this, we suggest that research on sociocultural processes of ability in PETE is needed as a first step to explore and disrupt dominant notions of ability and the purpose of 
PE. However, literature exploring PETE agents' views on ability is limited. Empirical work has been conducted in closely related contexts, such as Physical Activity and Sport Science programmes1 (e.g. Nabaskues et al., 2019), as well as studies that focused on interrelated themes such as embodied physicality (e.g. Wrench and Garrett, 2015). This research has contributed to uncovering future educators' narrow notions of ability in PE as a consequence of successful PE experiences and dominant discourses embedded in higher education programmes. Therefore, there is a need for continued effort along this line of inquiry as it might assist PE teacher educators to comprehend and build on preservice teachers' beliefs to disrupt and transform internalized embodied experi- ences in movement contexts (Brown, 2005).

Thus, if higher education is an appropriate space to challenge future educators' beliefs (Barber, 2018; Brown, 2005), then PETE should become a site for transformation to assist students to critically reflect on sociocultural processes and social justice issues embedded within PE (Sparkes et al., 2019; Walton-Fisette and Sutherland, 2018). However, in this review, implications for PETE were only raised by one article (Domangue and Solmon, 2012). In line with Walton-Fisette and Sutherland (2018), we recommend that the practical implications suggested for PE should be also applied to PETE programmes. Moreover, we acknowledge that pedagogical strategies in the lit- erature concerned with addressing social justice and sociocultural issues in PETE (for example, using the contextual twist and case studies to promote dialogue or asking students to look inwards to explore how their own life histories influence their belief systems) might be useful in chal- lenging narrow understandings of ability in PE, as this is a closely related field of study (e.g. Walton-Fisette et al., 2018).

Although all the reviewed papers strongly criticized traditional notions of ability, only Croston and Hills (2017) suggested the concept of physical literacy (Whitehead, 2010) as an alternative standpoint to think differently about ability. However, this philosophical perspective has received some criticism recently (Smith, 2011; Standal, 2015). This review has raised concerns about the idea of 'ability as physical literacy' (Wright and Burrows, 2006: 279), as this might continue to support a hierarchical order of students' capabilities, as it reinforces the quantitative attributes of performed movements (Standal, 2015). This is not to suggest that the concept of physical literacy is unhelpful. Rather, it is to warn scholars of the need to reconsider assumptions about ability.

With these thoughts in mind, and as reported by Standal (2015), we argue that the phenomenological concept of critical movement literacy offers an interesting framework to think about ability in a more flexible way. In fact, looking at ability as movement literacy, the notion of ability as something you could have more or less of is confronted as the focus is placed on learners' individual subjective experience in the movement context. Interestingly, this concept is grounded in a number of sociocultural, post-structural, feminist, philosophical, phenomenological and educational theories. This is an example of how a process of (in)compatibility across discourse communities - that is, connections between theories with similar 'cargo' - could lead to a useful cumulative and strengthened understanding of ability, and ultimately, contribute to building a maturing field of interest (Tinning, 2015), in this particular case, enlightening an alternative way to think about ability in PE. At this point it is worth mentioning that even though part of the rationale for this research is its increased theoretical scope, it could be perceived as a potential weakness to use Evans', Bourdieu's and 
other closely related concepts as search terms (e.g. physical capital or physicality). However, these search terms were introduced by the operator OR, so it does not exclude other theoretical perspectives that might be used for exploring notions of ability in PE.

Furthermore, movement literacy is presented as a pedagogical model that offers practical guidance to build a habitable learning space in PE (Standal, 2015), some of which is in line with several recommendations identified in this review. Yet, empirical research exploring how pedagogical principles of movement literacy might contribute to dealing with the heterogeneity among students in PE is scarce. Accordingly, we suggest future research should focus on how the

philosophy behind movement literacy might influence PETE and PE agents' way of thinking about ability and the purpose of PE.

Most participants in the reviewed articles were able-bodied male agents. In accordance with previous research (Domangue and Solmon, 2012; Hay and Macdonald, 2010b), we argue that attending to the experiences of 'not-so-able' participants might bring new insights to the recon- ceptualization of ability in PE. For instance, Grenier et al. (2014) analyzed the impact of having a teacher with a disability on students' perceptions about disability and PE. Taking the capability approach as a theoretical framework, which is focused on what the person can do, it was found that having a teacher with a physical impairment influenced pupils' normativity discourses as they began to overcome embedded prejudices grounded on the medical model and started to recognize different ways of participating in PE. This is argued to be an essential step for change, as con- sideration of different movement possibilities encourages individuals to reflect on the rules of the playing field (Shilling, 2004), which ultimately might broaden pupils' tolerance towards diversity (Evans et al., 2015).

Additionally, in line with Standal (2015), the usefulness of the capability approach as a frame to deal with difference among PE students has been supported, as it is considered an appropriate standpoint to create a learning environment where everybody has the capacity to move at their own level (Grenier et al., 2014). In this vein, we argue that the use of the capability approach in combination with sociocultural and post-structural lenses encountered in the present review might offer an interesting frame to uncover critical questions and transform the field.

\section{Conclusions}

The aims of this review were: (a) to systematically review empirical research on sociocultural processes of ability in PE and PETE from 2004 to 2019; (b) to explore theoretical frameworks that have been employed for that aim; and (c) to critically analyze practical implications suggested in order to think about ability in a more appropriate way to deal effectively with challenges of inclusion and equity.

Our findings suggest that despite the fact that Evans (2004) articulated ability as a social construct more than a decade ago, traditional discourses of ability in PE are still often uncontested. While the key findings from this review reinforce those of Wilkinson et al. (2013), this systematic review is innovative for several reasons. First, it contains and 
compiles the most recent research in the area. Second, it includes a further debate in relation to the field of PETE. Third, it comprises the key practical implications suggested. Fourth, it analyses the variety of theoretical lenses that have been used to make sense of empirical data. The diverse but inter-connected frameworks (i.e. sociocultural and post-structuralism) underpinning previous research have been useful to elucidate the counter- productive consequences of legitimated habitus in PE. Consequently, the field of PE continues to be a source of inequity and alienation for pupils who do not bring the valued dispositions to the field, which, in turn, limits any possibility to celebrate individual differences in daily practice.

The reviewed articles have acknowledged the importance of understanding ability as physical literacy, as well as recognizing a wide set of alternative capabilities. This is evident in the practical implications outlined above, which were directed mainly to PE teachers and students. However, suggestions for PETE programmes were limited. In considering the findings from this study, three major reflections are presented, with regards to: (a) the encountered gap in field of PETE; (b) the usefulness of looking at ability as movement literacy; and (c) the need to attend to the diversity of voices. These reflections are followed by suggestions for future research.

We argue that in an attempt to move the field forward, it is imperative for PE and PE teacher educators to critically reflect on how dominant discourses that reproduce narrow versions of ability might be challenged through daily pedagogical practice. In that direction, practical implications summarized in this review might be of consideration both for PE and PE teacher educators. Moreover, there is a need to increase the presence of social justice and sociocultural issues in teacher education programmes so that future educators are provided with a space to think about how individual differences might be addressed in PE. For that aim, we advocate for the incor- poration of pedagogical implications for PETE raised by Walton-Fisette et al. (2018). However, these steps require teacher educators and policy makers to rethink the resources and structure of PETE programmes. It is hoped that findings from this review might provide some basis to (re)direct academics' and policy makers' attention towards this field of interest.

In conclusion, evidence from practice reveals that policy expectations in relation to inclusion are not being met consistently in PE, as the way the field is structured does not provide equal learning opportunities for all. That implies a shift in philosophy, going beyond strategies of adaptation and expanding notions of physicality in order to challenge relations of power and common practices in PE. By doing so, stakeholders might be looking at ability from a more appropriate standpoint from which to provide habitable (Standal, 2015) spaces in PE - that is, learning environments capable of ensuring a safe space to enjoy movement, to be successful and experience agency to a higher number of embodied subjectivities in PE.

\section{Declaration of Conflicting Interests}

The author(s) declared no potential conflicts of interest with respect to the research, authorship, and/or publication of this article. 
Funding

The author(s) received no financial support for the research, authorship, and/or publication of this article.

ORCID iD

Itsaso Nabaskues-Lasheras https://orcid.org/0000-0003-1716-3343

Note

1. The Physical Activity and Sport Science programme is a four-year bachelor's degree, which is compulsory to access the one-year master's in PETE in the context where the study was conducted.

\section{References}

Barber W (2018) Inclusive and accessible physical education: Rethinking ability and disability in pre-service teacher education. Sport, Education and Society 23(6): 520-532.

Beltra'n-Carrillo VJ and Dev'is-Dev'is J (2019) El pensamiento del alumnado inactivo sobre sus experiencias negativas en educacio'n f'isica: los discursos del rendimiento, salutismo y masculinidad hegemo'nica. Revista Internacional de Ciencias del Deporte 15(55): $20-34$.

Bourdieu P (1990) The Logic of Practice. Cambridge: Polity Press.

Brown D (2005) An economy of gendered practices? Learning to teach physical education from the perspec- tive of Pierre Bourdieu's embodied sociology. Sport, Education and Society 10(1): 3-23.

Coates JK (2012) Teaching inclusively: Are secondary physical education student teachers sufficiently prepared to teach in inclusive environments? Physical Education and Sport Pedagogy 17(4): 349-365.

Croston A and Hills LA (2017) The challenges of widening 'legitimate' understandings of ability within physical education. Sport, Education and Society 22(5): 618-634.

Domangue EA and Solmon MA (2012) Fitness testing: How do students make sense of the gender disparities?

Sport, Education and Society 17(2): 207-224.

Dowling F (2011) 'Are PE teacher identities fit for postmodern schools or are they clinging to modernist notions of professionalism?' A case study of Norwegian PE teacher students' emerging professional identities. Sport, Education and Society 16(2): 201-222. 
Evans J (2004) Making a difference? Education and 'ability' in physical education. European Physical Education Review 10(1): 95-108.

Evans J (2014) Equity and inclusion in physical education PLC. European Physical Education Review 20(3): 319-334.

Evans J and Davies B (2017) In pursuit of equity and inclusion: Populism, politics and the future of educa- tional research in physical education, health and sport. Sport, Education and Society 22(5): 684-694.

Evans J and Penney D (2008) Levels on the playing field: The social construction of physical 'ability' in the physical education curriculum. Physical Education and Sport Pedagogy 13(1): $31-47$.

Evans AB, Brown LJ and Bright JL (2015) Non-disabled secondary school children's lived experiences of a wheelchair basketball programme delivered in the East of England. Sport, Education and Society 20(6): 741-761.

Felis-Anaya M, Martos-Garcia D and Dev'1s-Dev'1s J (2017) Socio-critical research on teaching physical education and physical education teacher education: A systematic review. European Physical Education Review 24(3): 314-329.

Fitzgerald H (2005) Still feeling like a spare piece of luggage? Embodied experiences of (dis) ability in physical education and school sport. Physical Education and Sport Pedagogy 10(1): 41-59.

Fitzgerald H (2012) 'Drawing' on disabled students' experiences of physical education and stakeholder responses'. Sport, Education and Society 17(4): 443-462.

Flintoff A, Fitzgerald H and Scraton S (2008) The challenges of intersectionality:

Researching difference in physical education. International Studies in Sociology of Education 18(2): 73-85.

Grant MJ and Booth A (2009) A typology of reviews: An analysis of 14 review types and associated methodologies. Health Information and Libraries Journal 26(2): 91-108.

Grenier MA, Horrell A and Genovese B (2014) Doing things my way: Teaching Physical Education with a disability. Adapted Physical Activity Quarterly 31(4): 325-342.

Hay PJ (2005) Making judgements - student ability and assessment in physical education. Journal of Physical Education New Zealand 38(1): 41-50.

Hay PJ and lisahunter (2006) 'Please Mr Hay, what are my poss(abilities)?': Legitimation of ability through physical education practices. Sport, Education and Society 11(3): 293-310.

Hay PJ and Macdonald D (2010a) Evidence for the social construction of ability in physical education. Sport, Education and Society 15(1): 1-18.

Hay PJ and Macdonald D (2010b) The gendering of abilities in Senior PE. Physical Education and Sport Pedagogy 15(3): 271-285.

Hennink M, Hutter I and Bailey A (eds) (2011) Textual data analysis. In: Qualitative Research Methods. 
London: SAGE, 233-268.

Hills L (2007) Friendship, physicality, and physical education: An exploration of the social and embodied dynamics of girls' physical education experiences. Sport, Education and Society 12(3): 317-336.

Hooks B (1982) Ain’t I a Woman? Black Women and Feminism. Boston: South End Press.

Hunter L (2004) Bourdieu and the social space of the PE class: Reproduction of doxa through practice. Sport, Education and Society 9(2): 175-192.

Koca C, Atencio M and Demirhan G (2009) The place and meaning of the field of PE in Turkish young people' s lives: A study using Bourdieu's conceptual tools. Sport, Education and Society 14(1): 55-75.

Moher D, Liberati A, Tetzlaff J, et al. (2009) Preferred Reporting Items for Systematic Reviews and Meta- Analyses: The PRISMA statement. PLos Med 6(7): e1000097.

Nabaskues I, Usabiaga O, Martos-Garcia D, et al. (2019) Comprensio'n de la habilidad desde la perspectiva del futuro profesorado de Educacio'n F'1sica. Retos 36(2): 118-124.

Nyberg G, Backman E and Larsson H (2019) Exploring the meaning of movement capability in physical education teacher education through student voices. European Physical Education Review.

Overton H, Wrench A and Garrett R (2017) Pedagogies for inclusion of junior primary students with disabilities in PE. Physical Education and Sport Pedagogy 22(4): 414-426.

Penney D, Jeanes R, O’Connor J, et al. (2018) Re-theorising inclusion and reframing inclusive practice in physical education. International Journal of Inclusive Education 22(10): 1062-1077.

Phoenix A and Pattynama P (2006) Editorial: Intersectionality. European Journal of Women's Studies 13(3): 187-192.

Redelius K and Hay P (2009) Defining, acquiring and transacting cultural capital through assessment in physical education. European Physical Education Review 15(3): 275-294.

Redelius K, Fagrell B and Larsson H (2009) Symbolic capital in physical education and health: To be, to do or to know? That is the gendered question. Sport, Education and Society 14(2): 245-260.

Saldaña J (2009) The Coding Manual for Qualitative Researchers. London: SAGE.

Serra P (2016) La perspectiva de ge'nero en los estudios de ciencias de la actividad f' 1 sica y el deporte. PhD Thesis, Universitat de Barcelona, Spain.

Shilling C (2004) Physical capital and situated action: A new direction for corporeal sociology. British Journal of Sociology of Education 25(4): 473-487.

Smith W (2011) Skill acquisition in physical education: A speculative perspective. Quest 63(3): 265-274. 
Sparkes AC, Martos-Garcia D and Maher AJ (2019) Me, osteogenesis imperfecta, and my classmates in physical education lessons: A case study of embodied pedagogy in action. Sport, Education and Society 24(4): 338-348.

Standal ØF (2015) Phenomenology and Pedagogy in Physical Education. London: Routledge.

Standal ØF and Aggerholm K (2016) Habits, skills and embodied experiences: A contribution to philosophy of physical education. Sport Ethics and Philosophy 10(3): 269282.

Svendby EB (2016) (Re)Telling lived experiences in different tales: A potential pathway in working towards an inclusive PE. Sport, Education and Society 21(1): 62-81.

Svennberg L (2017) Swedish PE teachers' understandings of legitimate movement in a criterion-referenced grading system. Physical Education and Sport Pedagogy 22(3): 257-269.

Tiden A, Redelius K and Lundvall S (2015) The social construction of ability in movement assessment tools.

Sport, Education and Society 22(6): 697-709.

Tinning R (2015) Commentary on research into learning in physical education: Towards a mature field of knowledge. Sport, Education and Society 20(5): 676-690.

Van Amsterdam N, Knoppers A, Claringbould I, et al. (2012) 'It's just the way it is .. . ' or not? How physical education teachers categorise and normalise differences. Gender and Education 24(7): 783-798.

Van Doodewaard C, Knoppers A and van Hilvoorde I (2018) 'Of course I ask the best students to demonstrate': Digital normalizing practices in physical education. Sport, Education and Society 23(8): 786-798.

Walton-Fisette JL and Sutherland S (2018) Moving forward with social justice education in physical educa- tion teacher education. Physical Education and Sport Pedagogy 23(5): 461468.

Walton-Fisette JL, Philpot R, Phillips S, et al. (2018) Implicit and explicit pedagogical practices related to sociocultural issues and social justice in physical education teacher education programs. Physical Edu- cation and Sport Pedagogy 23(5): 497-509.

Whitehead M (2010) Physical Literacy: Throughout the Lifecourse. New York: Routledge.

Wilkinson SD and Penney D (2016) The involvement of external agencies in extra-curricular physical education: Reinforcing or challenging gender and ability inequities? Sport, Education and Society 21(5): 741-758.

Wilkinson S, Littlefair D and Barlow-Meade L (2013) What is recognised as ability in physical education? A systematic appraisal of how ability and ability differences are socially constructed within mainstream secondary school physical education. European Physical Education Review 19(2): 147-164. 
Wiltshire G, Lee J and Evans J (2017) 'You don't want to stand out as the bigger one': Exploring how PE and school sport participation is influenced by pupils and their peers. Physical Education and Sport Pedagogy 22(5): 548-561.

Wrench A and Garrett R (2015) PE: It's just me: physically active and healthy teacher bodies. International Journal of Qualitative Studies in Education 28(1): 72-91.

Wright J and Burrows L (2006) Re-conceiving ability in physical education: A social analysis. Sport, Education and Society 11(3): 275-291. 
